\title{
Sleep Induced Hypoxemia in Chronic Obstructive Pulmonary Disease
}

\author{
Jing Zhang, ${ }^{1}$ Yan Wang, ${ }^{1}$ Jing Feng, ${ }^{1}$ Xin Sun ${ }^{2}$ \\ 'Respiratory Department of Tianjin Medical University General Hospital, Tianjin 300052, China, ${ }^{2}$ Respiratory Department of \\ Tianjin Haihe Hospital, Tianjin 300350, China.
}

\section{ABSTRACT}

Chronic obstructive pulmonary disease is a leading cause of morbidity and mortality worldwide. Sleep-induced hypoxemia is defined as "an $\mathrm{SpO}_{2}$ (oxyhemoglobin saturation) during sleep of < $90 \%$ for more than five minutes with a nadir of at least $85 \%$ " or " $>30 \%$ of total sleep time with an $\mathrm{SpO}_{2}$ of $<90 \%$ " in subject with a baseline awake $\mathrm{SpO}_{2}$ of $\geq 90 \%$. Patients with moderate or severe COPD run a high risk of developing SIH mainly because of alveolar hypoventilation and ventilationperfusion mismatch. Compared to their non-SIH brethren, SIH COPD patients have greater degrees of pulmonary hypertension and cor pulmonale, require more frequent hospitalizations, and sustain higher mortality rates. And the necessity of treatment of isolated SIH in COPD has been debated for years. In this mini review, the definition, reasons, prevalence, clinical significance and treatment approaches of SIH in COPD are summarized.

Keywords: chronic obstructive pulmonary disease; polysomnography; rapid eye movement; sleep-induced hypoxemia.

\section{INTRODUCTION}

Chronic obstructive pulmonary disease (COPD) is characterized by persistent airflow limitation that is usually progressive and is a leading cause of morbidity and mortality worldwide. ${ }^{1}$ When pulmonary function deteriorates, and when the disease progresses, the risk of alveolar hypoxia and consequent hypoxemia in the patients with COPD increases. Hypoxia plays an important role in the progression of COPD and the extrapulmonary comorbidities. Previous studies found even patients with COPD with mild awake hypoxemia who did not qualify for home oxygen therapy had significant nocturnal desaturation. The phenomenon of nocturnal hypoxemia complicating COPD has been recognized for at least 50 years. ${ }^{2}$ Increased sleep latency, decreased total sleep time, decreased slowwave sleep, decreased rapid eye movement (REM) sleep, and the most important, sleep-induced hypoxemia $(\mathrm{SIH})$, have been noted in many polysomnographic studies of patients with COPD. ${ }^{3} \mathrm{SIH}$ may predispose to pulmonary hypertension, cardiac arrhythmias during sleep and nocturnal death during exacerbations in patients with COPD. However, its importance and significance is not fully established.

\section{Definition OF SIH}

SIH in the ICSD-2 (The International Classification of Sleep Disorders: Diagnostic and Coding Manual) is defined as "an $\mathrm{SpO}_{2}$ (oxyhemoglobin saturation) during sleep of $<90 \%$ for more than five minutes with a nadir of at least $85 \%$ " or " $>30 \%$ of total sleep time with an $\mathrm{SpO}_{2}$ of $<90 \%$ " in subject with a baseline awake $\mathrm{SpO}_{2}$ of $\geq 90 \%$. $^{4,5}$

Correspondence: Jing Feng, Respiratory Department of Tianjin Medical University General Hospital, Tianjin 300052, China. Email: jing. feng2@duke.edu, Phone: 81100060. 


\section{Reasons of SIH in COPD}

Patients with COPD run a high risk of developing nocturnal hypoxemia. ${ }^{6,7}$

First, SIH occurs in patients with COPD independent of any changes in upper airway mechanics for a number of factors, mainly including: airway obstruction, hyperinflation, respiratory muscle dysfunction, blunted ventilatory responses to hypoxia and ventilationperfusion mismatch. Alveolar hypoventilation, a normal feature during sleep, has a disproportionate effect on hypoxemic patients because of their position on the oxyhemoglobin dissociation curve, leading to significant nocturnal desaturation, even in patients with mild awake hypoxemia. ${ }^{8}$ Alveolar hypoventilation appears to play a major role, especially during REM sleep. This was demonstrated by Becker ${ }^{9}$ in a study of nine patients with underlying COPD. Compared with wakefulness, minute ventilation decreased $16 \%$ during non-REM sleep and $32 \%$ during REM sleep, predominantly because of a decrease in tidal volume, measured with a pneumotachograph.

The second, arterial hypoxemia alone may be the product of worsening ventilation-perfusion mismatch with greater effective shunt. 5,10,11 In addition, loss of accessory muscle activity during sleep especially REM sleep result in a decreased functional residual capacity (FRC) and a deterioration in pulmonary gas exchange, which leads to worsening ventilation-perfusion relationships and also aggravates nocturnal hypoxemia.

Alveolar hypoventilation occurs predominantly during REM sleep. Ventilation-perfusion mismatch can occur during non-REM sleep episodes as well. ${ }^{6}$ Overall, tidal volume and minute ventilation are decreased during REM, but the breathing pattern during REM sleep is irregular, with sudden changes in respiratory amplitude and frequency. ${ }^{12}$

\section{Prevalence and Clinical Characteristics of SIH in COPD}

The prevalence of $\mathrm{SIH}$ among patients with COPD is unknown. Obviously, the lower the baseline $\mathrm{SpO}_{2}$ in the population studied the higher the prevalence of $\mathrm{SIH}$ is likely to be. There is limited information regarding the extent to which patients with COPD with mild to moderate daytime hypoxemia not qualifying for longterm oxygen therapy (LTOT) experience transient nocturnal desaturation.

Fletcher $^{13}$ showed that $27 \%$ of 135 patients with COPD with awake $\mathrm{PaO}_{2}$ exceeding $60 \mathrm{~mm} \mathrm{Hg}$ had $\mathrm{SIH}$. Although patients with REM-associated SIH had a lower awake $\mathrm{PaO}_{2}$ and higher awake $\mathrm{PaCO}_{2}$, these parameters were not predictive in identifying patients with $\mathrm{SIH}$.
Subsequently, several small studies of patients with COPD reported the prevalence of significant nocturnal desaturation to be approximately $50 \%$ in patients who had some degree of daytime hypoxemia. ${ }^{14,15}$ Similarly, after screening more than 800 patients with COPD, Lewis et al $^{16}$ found nocturnal desaturation in 29 of 59 (49\%) patients with a daytime SpO2<95\%. In another study, up to $25 \%$ of patients with COPD exhibit $\mathrm{SIH}$, despite having a daytime $\mathrm{PaO}_{2}$ above $60 \mathrm{~mm} \mathrm{Hg.}{ }^{17}$ There were two large studies of patients with COPD to assess the prevalence of nocturnal desaturation in 2011. Lacasse et al ${ }^{18}$ found that a significant proportion (38\%) of patients with moderate-to-severe COPD who did not qualify for home oxygen therapy based on their daytime $\mathrm{PaO}_{2}$ had nocturnal oxygen desaturation without evidence of sleep apnea. This study found that nocturnal desaturation without sleep apnea could not be predicted by any patient characteristic or physiological measure and Home oximetry was an effective practical method for screening this population. Another study included a wide range of unselected individuals with COPD and reported that 20 of $103(19.4 \%)$ patients had significant nocturnal desaturation. ${ }^{19}$ Results from this study suggested that monitoring oxygen saturation changes during a 6 min walk test was useful in helping to identify COPD patients who would experience significant nocturnal desaturation.

$\mathrm{KoO}^{20}$ found a mean decrease in $\mathrm{PaO}_{2}$ of $13.5 \mathrm{~mm} \mathrm{Hg}$ and a mean increase of $\mathrm{PaCO}_{2}$ of $8.3 \mathrm{~mm} \mathrm{Hg}$ during REM sleep. In a study of 16 COPD patients, ${ }^{3}$ the mean $\mathrm{SaO}_{2}$ was $90 \pm 6 \%$ and the lowest $\mathrm{SaO}_{2}$ during the night was $83 \pm 8 \%$. The percentage total sleep time (TST) with $\mathrm{a} \mathrm{SaO}_{2}$ less than $90 \%$ was $37 \pm 45 \%$. In another study, $\mathrm{PaCO}_{2}$ increases 3 to $10 \mathrm{~mm} \mathrm{Hg}$, and $\mathrm{PaO}_{2}$ decreases 2 to $8 \mathrm{~mm} \mathrm{Hg}{ }^{12}$

Compared to their nonhypoxemic brethren, hypoxemic COPD patients have greater degrees of pulmonary hypertension and cor pulmonale, require more frequent hospitalizations, and sustain higher mortality rates.

Pulmonary hypertension has been associated with $\mathrm{SIH}^{21}$ The increase in pulmonary artery pressure was associated with the decrease in $\mathrm{PaO}_{2}$ and less so with the increase in $\mathrm{PaCO}_{2}$. Profound hypoxemia and hypercapnia can occur in REM sleep and contribute to the development of cor pulmonale. ${ }^{22}$ Pulmonary vascular remodelling in COPD is the main cause of increase in pulmonary artery pressure and is thought to result from the combined effects of hypoxia, inflammation and loss of capillaries in severe COPD. ${ }^{23}$

Sergi ${ }^{24}$ found that $\mathrm{SIH}$ in COPD patients may represent an independent risk factor for the development of chronic respiratory failure in COPD patients with daytime $\mathrm{PaO}_{2}$ $>60 \mathrm{~mm} \mathrm{Hg}$. Survival may also be affected by the 
presence of SIH in COPD. In a retrospective study of 169 patients with COPD, Fletcher ${ }^{25}$ noted significantly decreased survival among patients with SIH compared with those without. Multiple regression analysis showed that quality of sleep was the best predictor of quality of life in subjects with COPD. Increased efforts to diagnose and treat sleep problems, including measures to improve factors that adversely affect sleep should receive great attention in the daily management of these patients, ${ }^{26}$ but isolated nocturnal desaturation was not associated with impairment of sleep quality or daytime function. ${ }^{16}$

Systematic inflammation is positively activated by $\mathrm{SIH}$. Although nocturnal hypoxemia does not reduce exercise capacity or hand-grip strength in patients with mild/moderate COPD, its effect on maximal exercise diastolic blood pressure seems to depend on the degree of hypoxemia. In addition, there is a positive relationship between maximal inspiratory pressure and mean peripheral oxygen saturation during sleep, as well as evidence of pronounced inflammatory activation in patients with nocturnal hypoxemia. ${ }^{27}$

Patients with COPD demonstrated significantly worse results in terms of accident frequency in the simulated driving situation because of $\mathrm{SIH}^{28}$ and had a high prevalence of restless legs syndrome related with $\mathrm{SIH} .{ }^{29}$

\section{Treatment of isolated SIH in COPD: Is it necessary?}

The necessity of treatment of isolated SIH in COPD has been debated for lots of years. ${ }^{30}$ The specific effect of worsening of hypoxaemia during sleep is difficult to assess. Whatever the specific role of sleep-related oxygen desaturation in severely hypoxaemic COPD patients, such individuals are usually treated with LTOT according to usual criteria. ${ }^{31,32}$ Fletcher $^{33}$ found no improvement in mortality but did demonstrate a hemodynamic benefit when patients with normal daytime oxygenation but nighttime hypoxemia received oxygen during sleep. Oxygen therapy during sleep was found neither an improvement in survival despite a reduction in pulmonary artery pressures nor a delay in the time to prescription of continuous oxygen therapy. 25,33 And some other randomized controlled trials of nocturnal oxygen treatment failed to demonstrate any improvement in pulmonary hemodynamic. ${ }^{34}$ When LTOT is started, the oxygen flow is individually adjusted to increase $\mathrm{PaO}_{2}$ to $>60 \mathrm{~mm} \mathrm{Hg.}{ }^{32,35}$ Such a flow may be insufficient during sleep. American Thoracic Society guidelines ${ }^{32,36}$ for diagnosis and treatment of COPD recommended increasing oxygen flow by $1 \mathrm{~L} / \mathrm{min}$ during sleep in patients undergoing LTOT to prevent nocturnal oxygen desaturation. However, those recommendations were not supported by any formal study demonstrating frequency of nocturnal desaturation in COPD patients breathing oxygen. ${ }^{7,36,37}$ Because most patients did not exhibit overnight desaturation despite not increasing their LTOT prescription overnight, so, these results challenge the recommendation of routinely increasing overnight oxygen flow in patients receiving LTOT. ${ }^{38,39}$ If sleep-related hypoxaemia has no effect on the outcome of the disease, prescription of oxygen during sleep would be a waste of medical resources.

Nocturnal non-invasive positive pressure ventilation (NNIPPV) is recommended to treat severe, stable COPD remains controversial, although a subgroup of patients with hypercapnea and sleep-disordered breathing (SDB) seems most likely to respond favorably. ${ }^{40}$ In a study, rehabilitation with nocturnal NIPPV resulted in the 6-minute walk test and in the longest non-stop walk distance in improvements. Further significant

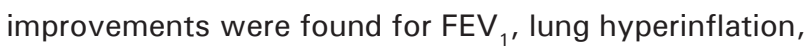
and blood gases in the NNIPPV treated, but not in the control subjects in advanced stage COPD. ${ }^{41}$ But a metaanalysis ${ }^{42}$ of clinical trials did not support the treatment of NNIPPV for isolated SIH in COPD. Another study ${ }^{43}$ did not furnish compelling evidence to change this point of view. McEvoy thought, compared with LTOT alone, NNIPPV resulted in a small improvement in survival. $\mathrm{FEV}_{1}$ and $\mathrm{PaCO}_{2}$ measured at 6 and 12 months were not different between groups. Patients assigned to NNIPPV + LTOT had reduced general and mental health and vigor. ${ }^{44}$

\section{Drugs for SIH in COPD}

Pharmacological therapies which ameliorate some of the factors contributing to hypoxemia during sleep described above might be an alternative approach. Previous studies support an improvement in lung function as an im portant mechanism of improvements in $\mathrm{SaO}_{2}$ during sleep. The Bronchodilators is the most commonly used drugs in patients with COPD. Oral theophylline therapy reduces the degree of air trapping in the lungs with consequent improvements in sleep $\mathrm{SaO}_{2}{ }^{45}$ Postma et al ${ }^{46}$ found that the nocturnal fall in $\mathrm{SaO}_{2}$ occurring in COPD patients was abolished by the B-agonist terbutaline in a slowrelease oral form, which was also associated withan improvement in FEV1. However B-Agonists in this form are now rarely used. Long-acting $B$-agonists (LABA) are a recommended part of care in patients with moderate/ severe COPD. The recent study ${ }^{47}$ assessed the effect of inhaled salmeterol on nocturnal sleeping arterial oxygen saturation and sleep quality and the findings indicated that addition of the inhaled LABA salmeterol improves $\mathrm{SaO}_{2}$ during sleep in patients with advanced COPD. Based on the pulmonary function testing results the authors proposed a reduction in hyperinflation as one likely underlying mechanism in this improvement. However, another double blind randomized study evaluated short term effect of a single evening dose 
of formoterol or tiotropium on the isolated nocturnal hypoxemia in a group of patients with moderate to severe stable COPD and diurnal $\mathrm{PaO}_{2}>60 \mathrm{mmHg}$ and reported that formoterol did not seem to influence the nocturnal hypoxemia in stable COPD patients probably for the worsening $\mathrm{V} / \mathrm{Q}$ ratio but tiotropium seemed to improved the nocturnal desaturations probably due to the reduction in the nocturnal bronchial cholinergic tone. ${ }^{48}$

There are ongoing controversies concerning the safety of long-acting B-agonists, and therefore the inhaled longacting anticholinergics may represent the best choice of therapy for SIH in COPD and the multicenter study assessed the effect of the long-acting anticholinergic agent tiotropium and reported a significant improvement in $\mathrm{SaO}_{2}$ during sleep, which was accompanied by an improvement in spirometry. ${ }^{49}$

More recently, as part of the National Emphysema Treatment Trial, lung volume reduction surgery has been shown to improve both sleep quality and nocturnal oxygenation in COPD. Although indications for performing an overnight polysomnogram in patients with COPD have been debated, recommendations have been presented. Future studies investigating disease mechanism and response to therapy in patients with sleep abnormalities and severe COPD are warranted. ${ }^{17}$

Since combined effects of inflammation, endothelial cell dysfunction, and angiogenesis appear to contribute to the development of pulmonary hypertension associated with COPD. Systemic vasodilators have not been found to be effective therapy. Selective pulmonary vasodilators including inhaled nitric oxide and phosphodiesterase inhibitors are promising treatments for patients with COPD associated pulmonary hypertension but further evaluation of these medications is needed prior to their routine use. ${ }^{50}$

Conventional hypnotics worsen nocturnal hypoxemia and, in severe cases, can lead to respiratory failure. As a recent medicine, melatonin can improve sleep in COPD. Further long-term studies involving larger number of patients are needed before melatonin can be safely recommended for the management of sleep disturbances in these patients. ${ }^{51}$

For systematic inflammtion, methylprednisolone in combination with conventional medical therapy can not only improve lung function values but also mean nocturnal oxyhemoglobin saturation and sleep duration in clinically stabilized COPD patients who experience nocturnal oxyhemoglobin desaturation. ${ }^{52}$

The prevalence of $\mathrm{SIH}$ in patients with COPD is high. Determination of SIH may be important because it may impact on sleep quality, extrapulmonary comorbidities and health status of life in COPD. Perhaps most clinically relevant, nocturnal oxygen desaturation is a marker of increased mortality in COPD. However the relevance of evaluating nocturnal hypoxemia in patients with COPD is still under debate. A large multi-center research should be necessary to evaluate the adverse consequence of $\mathrm{SIH}$ and the benefit of correcting isolated SIH on longterm mortality rates in patient's with COPD.

\section{REFERENCES}

1. GOLD Executive Committee. Global strategy for the diagnosis, management, and prevention of chronic obstructive pulmonary disease(UPDATED 2013). Available on http://www.goldcopd.org

2. Kardos P, Keenan J. Tackling COPD: a multicomponent disease driven by inflammation. MedGenMed. 2006;8(3):54.

3. Krachman SL, Chatila W, Martin UJ, Nugent T, Crocetti J, Gaughan J, Criner GJ. National Emphysema Treatment Trial Research Group. Effects of lung volume reduction surgery on sleep quality and nocturnal gas exchange in patients with severe emphysema. Chest. 2005;128(5):3221-8.

4. American Academy of Sleep Medicine. The international classification of sleep disorders: diagnostic and coding manual; 2nd ed. Westchester, IL: American Academy of Sleep Medicine. 2005;165-7.

5. Casey KR, Cantillo KO, Brown LK. Sleep-related hypoventilation/hypoxemic syndromes. Chest. 2007;131(6):1936-48.
6. Brijker F, van den Elshout FJ, Heijdra YF, Folgering HT. Underestimation of nocturnal hypoxemia due to monitoring conditions in patients with COPD. Chest. 2001;119(6):1820-6.

7. O'Donoghue FJ, Catcheside PG, Eckert DJ, McEvoy RD. Changes in respiration in NREM sleep in hypercapnic chronic obstructive pulmonary disease. J Physiol. 2004;559(Pt 2):663-73.

8. Catterall JR, Calverley PM, MacNee W, Warren PM, Shapiro CM, Douglas NJ, Flenley DC. Mechanism of transient nocturnal hypoxemia in hypoxic chronic bronchitis and emphysema. J Appl Physiol. 1985;59(6):1698-703.

9. Becker HF, Piper AJ, Flynn WE, McNamara SG, Grunstein RR, Peter JH, Sullivan CE. Breathing during sleep in patients with nocturnal desaturation. Am J Respir Crit Care Med. 1999;159(1):112-8.

10. Nikolaou E, Trakada G, Prodromakis E, Efremidis G, Pouli A, Koniavitou A, Spiropoulos K. Evaluation of arterial endothelin-1 levels, before and during a sleep study, in patients with bronchial asthma and chronic obstructive pulmonary disease. Respiration. 2003;70(6):606-10. 
11. Orem JM, Lovering AT, Vidruk EH. Excitation of medullary respiratory neurons in REM sleep. Sleep. 2005;28(7):801-7.

12. Krieger J. Breathing during sleep in normal subjects. In: Kryger MH, Roth T, Dement WC, editors. Principles and practices of sleep medicine. Philadelphia, PA: W.B. Saunders, $2005 ; 232-44$

13. Fletcher EC, Miller J, Divine GW, Fletcher JG, Miller T. Nocturnal oxyhemoglobin desaturation in COPD patients with arterial oxygen tensions above $60 \mathrm{~mm} \mathrm{Hg}$. Chest. 1987;92(4):604-8.

14. Zanchet RC, Viegas CA. Nocturnal desaturation: Predictors and the effect on sleep patterns in patients with chronic obstructive pulmonary disease and concomitant mild daytime hypoxemia. J Bras Pneumol. 2006;32(3):207-12.

15. Thomas VD, Kumar V, Gitanjali B. Predictors of nocturnal oxygen desaturation in chronic obstructive pulmonary disease in a South Indian population. J Postgard Med. 2002;48(2):101-4.

16. Lewis CA, Fergusson W, Eaton T, Zeng I, Kolbe J. Isolated nocturnal desaturation in COPD: prevalence and impact on quality of life and sleep. Thorax. 2009;64(2):133-8.

17. Krachman S, Minai OA, Scharf SM. Sleep abnormalities and treatment in emphysema. Proc Am Thorac Soc. 2008;5(4):536-42.

18. Lacasse Y, Sériès F, Vujovic-Zotovic N, Goldstein R, Bourbeau J, Lecours R, Aaron SD, Maltais F. Evaluating nocturnal oxygen desaturation in COPD--revised. Respir Med. 2011;105(9):1331-7.

19. Scott AS, Baltzman MA, Chan R, Wolkove N. Oxygen desaturation during a 6 min walk test is a sign of nocturnal hypoxemia. Can Respir J. 2011;18(6):333-7.

20. Koo KW, Sax DS, Snider GL. Arterial blood gases and pH during sleep in chronic obstructive pulmonary disease. Am J Med. 1975;58(5):663-70.

21. Minai OA, Pandya CM, Golish JA, Avecillas JF, McCarthy K, Marlow S, Arroliga AC. Predictors of nocturnal oxygen desaturation in pulmonary arterial hypertension. Chest. 2007;131(1):109-17.

22. McKenzie DK, Butler JE, Gandevia SC. Respiratory Muscle Function and Activation in Chronic Obstructive Pulmonary Disease (COPD). J Appl Physiol. 2009;107(2):621-9.

23. Chaouat A, Naeije R, Weitzenblum E. Pulmonary hypertension in COPD. Eur Respir J. 2008;32(5):1371-85.

24. Sergi M, Rizzi M, Andreoli A, Pecis M, Bruschi C, Fanfulla F. Are COPD patients with nocturnal REM sleep-related desaturations more prone to developing chronic respiratory failure requiring long-term oxygen therapy? Respiration. 2002;69(2):117-22.

25. Fletcher EC, Donner CF, Midgren B, Zielinski J, Levi-Valensi P, Braghiroli A, Rida Z, Miller CC. Survival in COPD patients with a daytime $\mathrm{PaO} 2$ greater than $60 \mathrm{~mm} \mathrm{Hg}$ with and without nocturnal oxyhemoglobin desaturation. Chest. 1992;101(3):649-55.
26. Nunes DM, Mota RM, de Pontes Neto OL, Pereira ED, de Bruin VM, de Bruin PF. Impaired sleep reduces quality of life in chronic obstructive pulmonary disease. Lung. 2009;187(3):159-63.

27. Mueller Pde T, Gomes MD, Viegas CA, Neder JA. Systemic effects of nocturnal hypoxemia in patients with chronic obstructive pulmonary disease without obstructive sleep apnea syndrome. J Bras Pneumol. 2008;34(8):567-74.

28. Orth M, Diekmann C, Suchan B, Duchna HW, Widdig W, Schultze-Werninghaus G, Rasche K, Kotterba S. Driving performance in patients with chronic obstructive pulmonary disease. J Physiol Pharmacol. 2008;59 Suppl 6:539-47.

29. Lo Coco D, Mattaliano A, Coco AL, Randisi B. Increased frequency of restless legs syndrome in chronic obstructive pulmonary disease patients. Sleep Med. 2009;10(5):572-6.

30. O'Reilly P, Bailey W. Long-term continuous oxygen treatment in chronic obstructive pulmonary disease: proper use, benefits and unresolved issues. Curr Opin Pulm Med. 2007;13(2):120-4.

31. Chaouat A, Weitzenblum E, Kessler R, Schott R, Charpentier C, Levi-Valensi P, Zielinski J, Delaunois L, Cornudella R, Moutinho dos Santos J. Outcome of COPD patients with mild daytime hypoxaemia with or without sleep-related oxygen desaturation. Eur Respir J. 2001;17(5):848-55.

32. American Thoracic Society. Standards for the diagnosis and care of patients with chronic obstructive pulmonary disease. Am J Respir Crit Care Med. 1995;152(5 Pt 2):S77-S121.

33. Fletcher EC, Luckett RA, Goodnight-White S, Miller CC, Qian W, Costarangos-Galarza C. A double-blind trial of nocturnal supplemental oxygen for sleep desaturation in patients with chronic obstructive pulmonary disease and a daytime $\mathrm{PaO} 2$ above 60 mm Hg. Am Rev Respir Dis. 1992;145(5):1070-6.

34. Chaouat A, Weitzenblum E, Kessler R, Charpentier C, Enrhart M, Schott R, Levi-Valensi P, Zielinski J, Delaunois L, Cornudella R, Moutinho dos Santos J. A randomized trial of nocturnal oxygen therapy in chronic obstructive pulmonary disease patients. Eur Respir J. 1999;14(5):1002-8.

35. Siafakas NM, Vermeire P, Pride NB, Paoletti P, Gibson J, Howard P, Yernault JC, Decramer M, Higenbottam T, Postma DS. Optimal assessment and management of chronic obstructive pulmonary disease (COPD). The European Respiratory Society Task Force. Eur Respir J. 1995;8(8):1398-420.

36. Plywaczewski R, Sliwinski P, Nowinski A, Kaminski D, Zieliński J. Incidence of nocturnal desaturation while breathing oxygen in COPD patients undergoing long-term oxygen therapy. Chest. 2000;117(3):679-83.

37. Kim V, Benditt JO, Wise RA, Sharafkhaneh A. Oxygen therapy in chronic obstructive pulmonary disease. Proc Am Thorac Soc. 2008;5(4):513-8.

38. Nisbet M, Eaton T, Lewis C, Fergusson W, Kolbe J. Overnight prescription of oxygen in long term oxygen therapy: time to reconsider the guidelines? Thorax. 2006;61(9):779-82. 
39. Croxton TL, Bailey WC. Long-term oxygen treatment in chronic obstructive pulmonary disease: recommendations for future research: an NHLBI workshop report. Am J Respir Crit Care Med. 2006;174(4):373-8.

40. Ozsancak A, D'Ambrosio C, Hill NS. Nocturnal noninvasive ventilation. Chest. 2008;133(5):1275-86.

41. Köhnlein T, Schönheit-Kenn U, Winterkamp S, Welte T, Kenn K. Noninvasive ventilation in pulmonary rehabilitation of COPD patients. Respir Med. 2009;103(9):1329-36.

42. Wijkstra PJ, Lacasse Y, Guyatt GH, Casanova C, Gay PC, Meecham Jones J, Goldstein RS. A meta-analysis of nocturnal noninvasive positive pressure ventilation in patients with stable COPD. Chest. 2003;124(1):337-43.

43. Clini E, Sturani C, Rossi A, Viaggi S, Corrado A, Donner CF, et al. The Italian multicentre study on noninvasive ventilation in chronic obstructive pulmonary disease patients. Eur Respir J. 2002;20(3):529-38.

44. McEvoy RD, Pierce RJ, Hillman D, Esterman A, Ellis EE, Catcheside PG, et al. Nocturnal non-invasive nasal ventilation in stable hypercapnic COPD: a randomised controlled trial. Thorax. 2009;64(7):561-6.

45. Mulloy E, McNicholas WT. Theophylline improves gas exchange during rest, exercise, and sleep in severe chronic obstructive pulmonary disease. Am Rev Respir Dis. 1993;148(4 Pt 1):1030-6.
46. Postma DS, Koeter GH, Mark TW, Reig RP, Sluiter HJ. The effects of oral slow-release terbutaline on the circadian variation in spirometry and arterial blood gas levels in patients with chronic airflow obstruction. Chest. 1985;87(5):653-7.

47. Ryan S, Doherty LS, Rock C, Nolan GM, McNicholas WT. Effects of Salmeterol on sleeping oxygen saturation in chronic obstructive pulmonary disease. Respiration. 2010;79(6):475-81.

48. Sposato B, Franco C. Short term effect of a single dose of formoterol or tiotropium on the isolated nocturnal hypoxemia in stable COPD patients: a double blind randomized study. Eur Rev Med Pharmacol Sci. 2008;12(3):203-11.

49. McNicholas WT, Calverley PM, Lee A, Edwards JC. Long-acting inhaled anticholinergic therapy improves sleeping oxygen saturation in COPD. Eur Respir J. 2004;23(6):825-31

50. Elwing J, Panos RJ. Pulmonary hypertension associated with COPD. Int J Chron Obstruct Pulmon Dis. 2008;3(1):55-70.

51. Nunes DM, Mota RM, Machado MO, Pereira ED, de Bruin VM, de Bruin PF. Effect of melatonin administration on subjective sleep quality in chronic obstructive pulmonary disease. Braz J Med Biol Res. 2008;41(10):926-31.

52. Sposato B, Mariotta S, Palmiero G, Ricci A, Gencarelli G, Franco C. Oral corticosteroids can improve nocturnal isolated hypoxemia in stable COPD patients with diurnal PaO2 > 60 mmHg. Eur Rev Med Pharmacol Sci. 2007;11(6):365-72. 